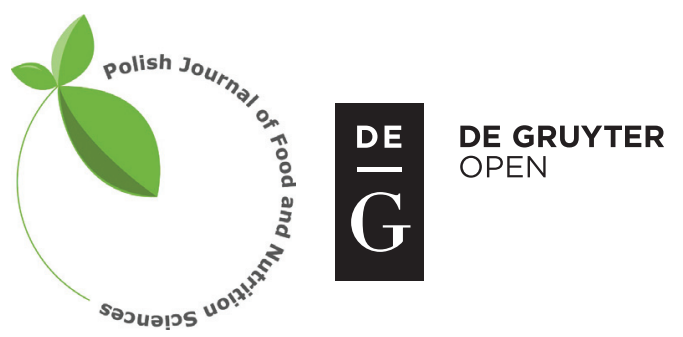

Pol. J. Food Nutr. Sci., 2016, Vol. 66, No. 3, pp. 179-188 DOI: $10.1515 /$ pjfns-2016-0006 http://journal.pan.olsztyn.pl

Original article

Section: Nutritional Research

\title{
Biological Activities and Nutraceutical Potentials of Water Extracts from Different Parts of Cynomorium coccineum L. (Maltese Mushroom)
}

\author{
Paolo Zucca ${ }^{1,2}$, Antonio Argiolas ${ }^{3}$, Mariella Nieddu $^{1}$, Manuela Pintus ${ }^{1}$, Antonella Rosa ${ }^{4}$, \\ Fabrizio Sanna ${ }^{3}$, Francesca Sollai ${ }^{1}$, Daniela Steri ${ }^{1}$, Antonio Rescigno ${ }^{1, *}$
}

\author{
'Dipartimento di Scienze Biomediche, Sezione di Biochimica, Biologia e Genetica, Università di Cagliari, Italy \\ ${ }^{2}$ Consorzio UNO, Oristano, Italy \\ ${ }^{3}$ Dipartimento di Scienze Biomediche, Sezione di Neuroscienze e Farmacologia Clinica, Università di Cagliari, Italy \\ ${ }^{4}$ Dipartimento di Scienze Biomediche, Sezione di Patologia, Università di Cagliari, \\ Cittadella Universitaria, 09042 Monserrato (CA), Italy
}

Key words: functional food, nutraceuticals, Cynomorium coccineum, Maltese Mushroom, tarthuth, B16F10 melanoma cells

Maltese Mushroom (Cynomorium coccineum L.) is a non-photosynthetic plant that has been used in traditional medicine for many centuries. In this paper, water extracts from the whole plant, external layer and peeled plant were studied to determine the main components responsible for its biological activities, i.e., its antimicrobial, antioxidant, and anti-tyrosinase activities; its cytotoxicity against mouse melanoma B16F10 cells; and its pro-erectile activity in adult male rats. The results of electron transfer and hydrogen transfer assays showed that the antioxidant activity was mainly due to anthocyanins in the external layer, whereas the external layer and peeled plant extracts both inhibited the microbial growth of several Gram-positive strains. In contrast, the whole plant extract had the highest anti-tyrosinase activity and exhibited pro-erectile activity when administered subcutaneously. Overall, this study elucidated which parts of Maltese Mushroom are responsible for its antimicrobial, antioxidant, and anti-tyrosinase activities and thus which extracts have potential for use in nutraceutical formulations.

\section{ABBREVIATIONS}

EL - water extract from the external layer of C. coccineum; PP - water extract from the peeled $C$. coccineum plant; and WP - water extract from the whole C. coccineum plant.

\section{INTRODUCTION}

Cynomorium coccineum $\mathrm{L}$. is a non-photosynthetic, parasitic plant of the Cynomoriaceae family that is ubiquitous in the Mediterranean basin and Arabian Peninsula [Dharmananda, 2011]. It is commonly known as "tarthuth" in Arabic countries or Maltese "Mushroom" in the western world [Cui et al., 2013; Dharmananda, 2011] because it does not have any chlorophyll (Figure 1, part (a)). The Cynomorium genus also includes $C$. songaricum, which is found in western Asia.

Of the two Cynomorium species, $C$. songaricum is the most well-known and has been widely studied because of traditional Chinese medicine. A comprehensive ethnopharmacological and phytochemical review on the Cynomorium genus in China was recently published [Cui et al., 2013]. The pharmaceutical and ethnobotanical properties of Maltese Mushroom are also

\footnotetext{
* Corresponding Author: Tel.: +39070 675 4516; Fax: +39070 6754527

E-mail: rescigno@unica.it (Prof. Antonio Rescigno)
}

known and were recorded centuries ago. As even reported in the Bible [Duke, 2008], it has been utilized in antihemorrhoidal, aphrodisiac, hypotensive, antiemetic, and antitumor applications and in the stimulation of spermatogenesis [Dharmananda, 2011; Wang et al., 2010; Yang et al., 2010; Yu et al., 2010]. During famines, it was eaten by humans, and it is currently used in herbal infusions and other food preparations [Cui et al., 2013; Jin et al., 2014].

Some of the biological activities of Maltese Mushroom have been studied in animal and in vitro models [Jin et al., 2014; Shi et al., 2011] and were recently reviewed [Cui et al., 2013; Jin et al., 2014; Meng et al., 2013]. In previous works, the lipid composition of the Maltese Mushroom fixed oil was detailed, and its biological activity towards intestinal epithelial cells was determined [Rosa et al., 2012, 2015]. Moreover, aqueous and methanolic extracts from Maltese Mushroom stems exhibited significant antifungal [Gonçalves et al., 2015] and antioxidant activities as demonstrated by several antioxidant assays and in vitro biological models [Zucca et al., 2013a]. In these extracts, the main polyphenols detected were gallic acid (3,4,5-trihydroxybenzoic acid, CAS 149-91-7) and cyanidin 3-O-glucoside (chrysanthemin or kuromanin, CAS 7084-24-4). Both of these compounds are well-known, promising food supplements [Jayamani \& Shanmugam, 2014; Mulabagal et al., 2012] because the biological activities of the polyphenolic fractions of the plant extracts 


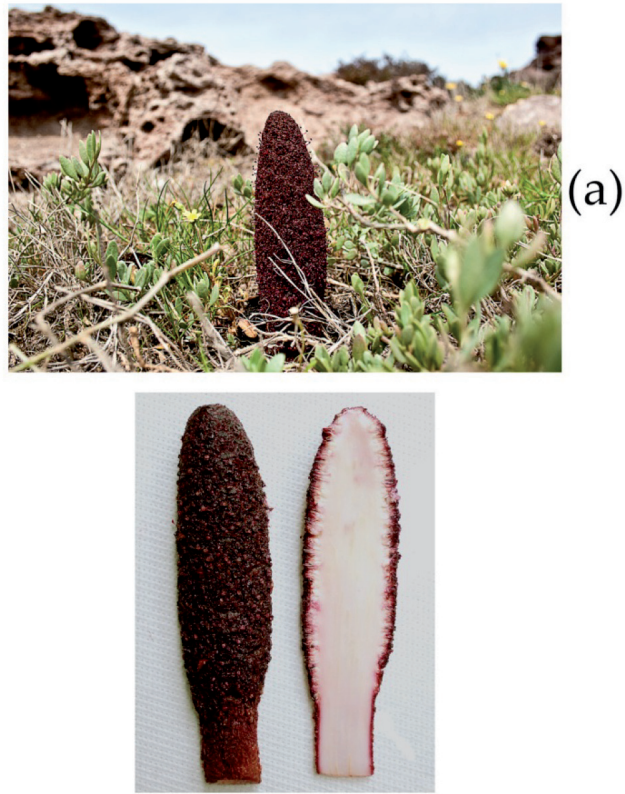

(b)<smiles>O=C(O)c1cc(O)c(O)c(O)c1</smiles>

(c)

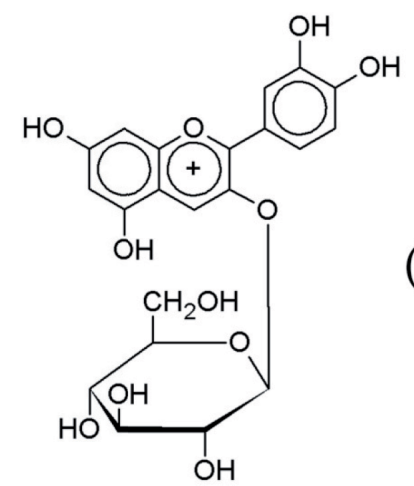

FIGURE 1. (a) A whole plant (WP) growing in a coastal area of Sardinia, Italy. (b) Slices of Maltese Mushroom (C. coccineum) showing the vividly colored external layer (EL) and the nearly colorless internal peeled part (PP). The two main constituents of the extracts were determined to be gallic acid (c) and cyanidin 3-O-glucoside (d); the former is nearly equally distributed between the external layer (EL) and peeled plant (PP), whereas the latter is nearly absent in the PP and highly concentrated in the EL, which is consistent with the intense color of anthocyanins.

are currently widely studied [Chahmi et al., 2015; Sanjust et al., 2008; Traka \& Mithen, 2011]. In particular, several studies focused on the composition of edible and medicinal plant extracts to determine their beneficial health effects and their potential use as a food supplement [Traka \& Mithen, 2011; Zucca et al., 2013a]. These reports demonstrate that Maltese Mushroom is a promising nutraceutical source.

Maltese Mushroom has a red-brown external layer (EL), whereas the peeled plant (PP) is nearly colorless (see Figure 1, part (b)). Gallic acid is nearly equally distributed between these two parts, whereas cyanidin 3-O-glucoside is nearly absent in the PP and highly concentrated in the EL [Zucca et al., 2013a], which is consistent with the intense color of anthocyanins.

Based on these observations, the water extracts from the whole plant (WP), external layer (EL) and peeled plant (PP) were studied to elucidate the roles of the main components. The biological activities (i.e., the cytotoxicity and antimicrobial, antioxidant, anti-tyrosinase and pro-erectile activities) of the extracts were tested to determine the specific plant parts that give rise to them. This work could potentially help in the formulation of Maltese Mushroom-based nutraceuticals; therefore, only aqueous extracts were included in this study.

\section{MATERIALS AND METHODS}

\section{Chemicals and instrumentation}

All reagents were of the best commercial grade available and were used without further purification. Analytical grade methanol, ethyl acetate, acetonitrile and $85 \% \mathrm{w} / \mathrm{w}$ phosphoric acid solvents were obtained from Sigma-Aldrich, Fluka (Milan, Italy). A cyanidin-3-O-glucoside standard was pur- chased from Extrasynthese (Genay, France). Ultrapure water $(18 \mathrm{~m} \Omega)$ was obtained with a Milli-Q Advantage A10 system (Millipore, Milan, Italy). Spectrophotometric measurements were performed using an UltroSpec 2100 pro instrument (Amersham Bioscience, Milan, Italy). Fatty acid methyl ester standards were purchased from Sigma-Aldrich (Milan, Italy). All solvents were of the highest available purity and were also purchased from Sigma-Aldrich. Methanolic $\mathrm{HCl}(3 \mathrm{~N})$ was purchased from Supelco (Bellefonte, PA). The fatty acid methyl ester content was measured using a Hewlett-Packard (HP) 6890 gas chromatograph (Hewlett-Packard, Palo Alto, USA) equipped with a flame ionization detector (FID) and a cyanopropyl methylpolysiloxane HP-23 FAME column (30 m x 0.32 mm x $0.25 \mu \mathrm{m})$ (Hewlett-Packard).

\section{Plant materials}

Maltese Mushroom was collected in April 2014 in Portoscuso (southwestern Sardinia, Italy), where it is known as "cagalloni strantaxiu". Stem reference materials (AR-CC-2014/4/1) were deposited in the collection of the Department of Biomedical Sciences at the University of Cagliari. The samples were carefully handled as described elsewhere [Zucca et al., 2013a]. In part of the samples, red-brown external layer (EL), colorless peeled plant (PP) (Figure 1, part (b)) were carefully separated from whole plant (WP), and the three samples were freeze-dried using Telstar LyoQuest -55 (Milan, Italy).

\section{Preparation of Maltese Mushroom extracts for antioxidant, anti-tyrosinase, antimicrobial and cytotoxicity experiments \\ Freeze-dried Maltese Mushroom plant material was} ground to a powder and extracted at room temperature. 
The material $(1 \mathrm{~g})$ was soaked in $5 \mathrm{~mL}$ of distilled water and mildly agitated for $60 \mathrm{~min}$. The supernatant was collected after centrifugation at $8000 \times g$ for $10 \mathrm{~min}$ at $4^{\circ} \mathrm{C}$. The procedure was repeated seven times until the supernatant was colorless. The total volume of the extracts was lyophilized and stored at $4^{\circ} \mathrm{C}$.

\section{Preparation and purification of Maltese Mushroom extracts for pro-erectile activity testing}

A slightly different extraction procedure was followed: $1 \mathrm{~g}$ of powdered plant material was dissolved in $10 \mathrm{~mL}$ of $\mathrm{H}_{2} \mathrm{O}$. After vortexing the mixture overnight, the resulting suspension was centrifuged at $8000 \times \mathrm{g}$ for $10 \mathrm{~min}$ at $4^{\circ} \mathrm{C}$. The supernatant was removed and stored at $4^{\circ} \mathrm{C}$. The remaining powder was dissolved in $10 \mathrm{~mL}$ of $\mathrm{H}_{2} \mathrm{O}$. After the mixture was vortexed for $1 \mathrm{~h}$ and then centrifuged at $8000 \times g$ for $15 \mathrm{~min}$ at $4^{\circ} \mathrm{C}$, the supernatant was removed, combined with the first supernatant and stored at $4^{\circ} \mathrm{C}$ until use (Extract Solution 1, ES-1).

ES-1 (10 mL) was passed twice through a Sep-Pak C18 cartridge (Waters Corporation, Milford, MA, USA) that had been washed with $10 \mathrm{~mL}$ of methanol and $10 \mathrm{~mL}$ of $\mathrm{H}_{2} \mathrm{O}$. Then the extract solution was collected and stored at $4^{\circ} \mathrm{C}$ until use (Extract Solution 2, ES-2). It should be noted that ES-2 was completely colorless. To some extent, ES-2 could be similar to the PP extract. The pigmented material adsorbed in the Sep-Pak C18 was then eluted with $10 \mathrm{~mL}$ of methanol, which was collected and evaporated under a nitrogen stream. The remaining material was then dissolved in $5 \mathrm{~mL}$ of saline to give a colored solution, which was stored at $4^{\circ} \mathrm{C}$ until use (Extract Solution 3, ES-3, that to some extent resembles the composition of the EL extract).

Animal experiments were performed according to the guidelines of the European Communities Directive of September 22, 2010 (2010/63/EU) and Italian legislation (D.L. March 4, 2014, n. 26) and were approved by the Ethical Committee for Animal Experimentation of the University of Cagliari.

\section{Nutritional facts label}

The nutritional facts were determined according to current legislation (Regulation EU No. 1169/2011 of the European Parliament and of the Council of 25 October 2011 on the provision of food information to consumers) at the facilities of "Laboratorio Chimico Merceologico della Sardegna" (Cagliari, Italy).

\section{Total phenolic, flavonoid, and anthocyanin content determination}

The total soluble phenolic content was determined using the Folin-Ciocalteu reagent as described in a previous report [Zucca et al., 2013a]. Gallic acid was used as the standard (linearity range $0.05-0.6 \mathrm{mmol} / \mathrm{L}$ ), and the results were expressed in gallic acid milliequivalents (mGAE).

A spectrophotometric method was used to quantify the total flavonoid content [Zucca et al., 2010a]. Briefly, $0.25 \mathrm{~mL}$ of the sample, $1.25 \mathrm{~mL}$ of $\mathrm{H}_{2} \mathrm{O}$, and $0.075 \mathrm{~mL}$ of $\mathrm{NaNO}_{2}$ $(5 \% \mathrm{w} / \mathrm{v})$ were incubated for $5 \mathrm{~min}$ at $25^{\circ} \mathrm{C}$. Then, $0.15 \mathrm{~mL}$ of $\mathrm{AlCl}_{3}(10 \% \mathrm{w} / \mathrm{v})$ was added to the mixture. After $6 \mathrm{~min}$,
$0.5 \mathrm{~mL}$ of $1 \mathrm{~mol} / \mathrm{L} \mathrm{NaOH}$ and $0.275 \mathrm{~mL}$ of $\mathrm{H}_{2} \mathrm{O}$ were also added, and the absorbance was measured at $510 \mathrm{~nm}$. Catechin was used as the standard (linearity range $0.1-0.6 \mathrm{mmol} / \mathrm{L}$ ), and the results were expressed in catechin milliequivalents (mCE) using a standard curve.

The total anthocyanin content was determined by a spectrophotometric assay based on differential $\mathrm{pH}$ absorbance [Delazar et al., 2010]. Briefly, the absorbances at $510 \mathrm{~nm}$ and $700 \mathrm{~nm}$ were measured at both $\mathrm{pH} 1.0$ and 4.5 using $0.2 \mathrm{~mol} / \mathrm{L} \mathrm{HCl} /$ $\mathrm{KCl}$ and $1 \mathrm{~mol} / \mathrm{L}$ sodium acetate buffers, respectively. The final absorbance was calculated using Equation 1.

$$
A_{\text {fin }}=\left[\left(A_{510}-A_{700}\right)_{p H 1.0}-\left(A_{510}-A_{700}\right)_{p H 4.5}\right]
$$

The molar extinction coefficient of cyanidin 3-O-glucoside $\left(29,300 \mathrm{M}^{-1} \cdot \mathrm{cm}^{-1}\right)$ was then used in the Lambert-Beer equation to calculate the total anthocyanin concentration (Equation $2, l=$ optical path).

$$
[\text { total anthocyanin }]=\frac{A_{\text {fin }}}{29,300 \mathrm{M}^{-1} \mathrm{~cm}^{-1} \cdot \mathrm{l}}
$$

\section{Antioxidant assays}

The 1,1-diphenyl-2-picrylhydrazyl (DPPH·) radical scavenging assay was performed according to a well-known protocol [Huang et al., 2005]. The results were expressed in Trolox milliequivalents (mTE, linearity range $5-50 \mu \mathrm{mol} / \mathrm{L}$ ) and as $\mathrm{IC}_{50}$ values.

The ferric reducing antioxidant power (FRAP) assay was performed as previously described [Huang et al., 2005]. Trolox was used for the calibration curve, and the results were expressed in Trolox milliequivalents (mTE) and in mmol of Fe(II) per gram of dry material (mmol FeII/g).

The Trolox equivalent antioxidant capacity (TEAC) assay was performed using the 2,2'-azinobis (3-ethylbenzothiazoline 6-sulfonate) (ABTS) cationic radical as previously described [Zucca et al., 2013a]. The results were expressed in Trolox milliequivalents (mTE, linearity range $0.1-0.8 \mathrm{mmol} / \mathrm{L}$ ) and as $\mathrm{IC}_{50}$ values.

The oxygen radical absorbance capacity-pyrogallol red (ORAC-PYR) assay was performed according to a recently described method [Zucca et al., 2010a]. The results were expressed in Trolox milliequivalents (mTE, linearity range $0.1-0.8 \mathrm{mmol} / \mathrm{L})$.

\section{Extraction and saponification of Maltese Mushroom oil}

Oil was extracted from the external layer (EL) and internal peeled part (PP) using the procedure of Folch et al. [1957] in which $12 \mathrm{~mL}$ of a 2:1 $(v / v) \mathrm{CHCl}_{3}: \mathrm{MeOH}$ solution was added to the powder sample. After filtration, $4 \mathrm{~mL}$ of $\mathrm{H}_{2} \mathrm{O}$ was added to the mixture, which was then centrifuged at $900 \times g$ for $1 \mathrm{~h}$ to separate the $\mathrm{CHCl}_{3}$ fraction (lipophilic extract) from the $\mathrm{MeOH} / \mathrm{H}_{2} \mathrm{O}$ mixture. Aliquots of the dried $\mathrm{CHCl}_{3}$ fractions were subjected to mild saponification [Rosa et al., 2015]. Aliquots of the dried fatty acids were then methylated with $1 \mathrm{~mL}$ of methanolic $\mathrm{HCl}(3 \mathrm{~N})$ [Rosa et al., 2015] for $30 \mathrm{~min}$ at room temperature. After $n$-hexane and $\mathrm{H}_{2} \mathrm{O}$ were added to the samples, they were centrifuged for $20 \mathrm{~min}$ at $900 \times g$. The hexane phase containing the fatty acid methyl 
esters was collected, and the solvent was subsequently evaporated. Then, the residue was dissolved in $n$-hexane, and aliquots of the samples were subjected to gas chromatography analysis (GC). All solvent evaporation procedures were performed under vacuum.

\section{GC analysis of the fatty acid methyl esters}

The fatty acid methyl ester content was measured using an HP 6890 system. Nitrogen was used as the carrier gas at a flow rate of $2 \mathrm{~mL} / \mathrm{min}$. The oven, injector, and detector temperatures were set to $175^{\circ} \mathrm{C}, 250^{\circ} \mathrm{C}$, and $300^{\circ} \mathrm{C}$, respectively. The fatty acid methyl esters were identified by comparing the observed retention times to those of standard compounds. The amounts of the individual fatty acids were calculated as percentages of the total amount of fatty acids ( $\mathrm{g} \%$ ) using the Hewlett-Packard A.05.02 software.

\section{Antimicrobial activity}

All bacterial strains used in this study belong to the Leibniz Institute DSMZ (German Collection of Microorganism and Cell Cultures). In particular, four Gram-negative and five Gram-positive reference bacterial strains were used: Staphylococcus aureus DSM 1104, methicillin-resistant Staphylococcus aureus (MRSA), Staphylococcus epidermidis DSM 1798, Enterococcus faecalis DSM 2570, Escherichia coli DSM 1103, Enterobacter cloacae DSM 30054T, Pseudomonas aeruginosa DSM 1117, Acinetobacter baumannii DSM 30007T, and Klebsiella pneumoniae DSM 681.

Inoculum suspensions were prepared using the direct colony suspension method according to CLSI procedures [Institute, 2012]. Saline suspensions of isolated colonies, which were selected from an 18-24 h Luria-Bertani agar plate, were used to achieve a turbidity equivalent to a 0.5 McFarland standard.

The antibacterial activities of the Maltese Mushroom extracts were determined using the disc diffusion method according to CLSI procedures [Institute, 2012]. Petri plates were prepared with $25 \mathrm{~mL}$ of Mueller-Hinton agar (MHA). The inoculum suspensions were swabbed on the MHA plate surface, and $1 \mathrm{mg}$ of dry extract was added to each disk separately. A negative control was prepared by placing a specific amount of Milli-Q water on the control disk surface. Gentamicin and oxacilline were used as positive controls.

\section{Tyrosinase inhibition}

Tyrosinase from Agaricus bisporus was purified as described previously [Sollai et al., 2008]. Experiments were performed to check for possible contaminant enzyme activities [Rescigno et al., 2007]. In particular, syringaldazine was used as the substrate [Zucca et al., 2011] to rule out laccase activity (<0.001 E.U./mL [Zucca et al., 2010b]) because contaminant laccases might share substrates, i.e., o-diphenols, with tyrosinase, leading to ambiguous results.

The tyrosinase activity was measured by monitoring the absorbance at $625 \mathrm{~nm}\left(\varepsilon_{625}=11,120 \mathrm{M}^{-1} \cdot \mathrm{cm}^{-1}\right)$ after the formation of an adduct between 4-tert-butyl-1,2-benzoquinone (TBBQ) and 4-amino- $N, N$-diethylaniline (ADA), as previously described [Rescigno et al., 1999; Zucca et al., 2013b]. The assay mixture, which had a final volume of $1 \mathrm{~mL}$, contained a $50 \mathrm{mmol} / \mathrm{L}$ sodium phosphate buffer at pH 7.0, 5 mmol/L 4-tert-butylcatechol (TBC), $0.75 \mathrm{mmol} / \mathrm{L}$ ADA and 2 E.U. of the enzyme (1 tyrosinase E.U. was defined as the amount of enzyme capable of producing $1 \mu \mathrm{mol}$ of the adduct between TBBQ and ADA per minute at $\mathrm{pH} 7$ and $25^{\circ} \mathrm{C}$ ). The tyrosinase inhibition by the test samples was quantified by the concentration necessary to achieve $50 \%$ inhibition $\left(\mathrm{IC}_{50}\right)$.

\section{Cell line and culture conditions}

The B16F10 mouse melanoma cell line (ICLC ATL 99010) was purchased from the National Institute for Cancer Research c/o CBA (Genoa, Italy). Subcultures of the cell line were grown in a $75 \mathrm{~cm}^{2}$ culture flask in DMEM medium supplemented with $10 \%$ fetal bovine serum (FBS), 2 mmol/L L-glutamine, $1 \%$ non-essential amino acids, $1 \mathrm{mmol} / \mathrm{L} \mathrm{Na}$ pyruvate, $100 \mathrm{U} / \mathrm{mL}$ penicillin and $100 \mu \mathrm{g} / \mathrm{mL}$ streptomycin at $37^{\circ} \mathrm{C}$ in $5 \% \mathrm{CO}_{2}$.

\section{MTT assay for cell viability}

The cytotoxic effects of the Maltese Mushroom water extracts (WP, PP, and EL) were evaluated by conducting the 3-(4,5-dimethylthiazol-2-yl)-2,5-diphenyltetrazolium bromide (MTT, CAS 298-93-1) assay, in which the tetrazolium salt is cleaved by mitochondrial dehydrogenases in viable cells [Rosa et al., 2012; Schiller et al., 1992], on the B16F10 mouse melanoma cells. MTT is a yellow, water-soluble tetrazolium salt. Metabolically active cells can convert the dye into a dark blue, water-insoluble formazan dye by reductively cleaving the tetrazolium ring. In brief, $3 \times 10^{3}$ cells $/ \mathrm{mL}$ in $100 \mu \mathrm{L}$ of medium were seeded into a 96 -well plate and incubated at $37^{\circ} \mathrm{C}$. After a $48 \mathrm{~h}$ incubation period, the aqueous extracts were added to the cultures in concentrations ranging from 25 to $500 \mu \mathrm{g} / \mathrm{mL}$, and the cells were incubated for an additional $24 \mathrm{~h}$ at $37^{\circ} \mathrm{C}$. The MTT solution $\left(8 \mu \mathrm{L}, 5 \mathrm{mg} / \mathrm{mL}\right.$ in $\left.\mathrm{H}_{2} \mathrm{O}\right)$ was then added to the culture, which was maintained at $37^{\circ} \mathrm{C}$ for $4 \mathrm{~h}$. The cells were lysed with $100 \mu \mathrm{L}$ of DMSO, and the color was measured at $570 \mathrm{~nm}$ with an auto microplate reader (Infinite 200, Tecan, Austria). The absorbance was proportional to the number of viable cells.

In addition, melanin content in the B16F10 cell cultures was determined by a previously described method [Soddu et al., 2004].

\section{Pro-erectile activity in adult male rats}

The effects of the Maltese Mushroom extracts (ES-1, ES-2, and ES-3) on penile erection in male rats were studied as described in the literature [Sanna et al., 2011, 2012]. Briefly, adult male Sprague-Dawley rats (225-250 g, Harlan Nossan, Italy) were housed at $24^{\circ} \mathrm{C}$ and $60 \%$ humidity and given water and standard laboratory food ad libitum. Aliquots of ES-1, its dilutions with saline, or ES-2 or ES-3 were administered subcutaneously (SC) in volumes of $0.1 \mathrm{~mL} / 80 \mathrm{~g}$ of rat body weight. Rats injected with the same volume of saline were used as controls. After treatment, each rat was placed in a Plexiglas cage $(30 \times 30 \times 30 \mathrm{~cm})$ by itself and observed for $60 \mathrm{~min}$ to count the number of penile erection episodes and monitor other gross behavioral changes. 


\section{Statistical analysis}

GraFit 7 (Erithacus Software, London, UK) and the R 2.5.1 software (R Foundation for Statistical Computing, Vienna) were used for the statistical analysis. All analyses were performed at least in triplicate unless otherwise stated. The statistical significance of differences was evaluated by analysis of variance (one-way ANOVA). Statistical evaluation of the penile erection experiments was performed using one-way ANOVA followed by Tukey's multicomparison test. For the B16F10 cell experiments, the statistical significance of differences was evaluated using one-way ANOVA followed by the Bonferroni post-test; the differences were considered to be significant at $P<0.001, P<0.01$, and $P<0.05$.

\section{RESULTS AND DISCUSSION}

\section{Nutritional facts of Maltese Mushroom}

Previous reports ascribe several biological activities to Maltese Mushroom [Rosa et al., 2012; Wang et al., 2010; Zucca et al., 2013a], suggesting that its extract has a potential for use in nutraceutical formulations. Based on the possibility that Maltese Mushroom might be used for human nutrition, the nutritional facts for the whole plant were collected. The results are reported in Table 1. Carbohydrates, including $27 \%$ dietary fibers, were the principal component (approximately 50\%). A significant portion of protein (approximately 9\%) was also detected, whereas only a small amount of lipids $(1 \%)$ were detected. Most of the lipids were unsaturated fats. Particularly, polyunsaturated fats, which are thought to be beneficial for many human pathological conditions [Roncaglioni et al., 2013], constituted nearly $40 \%$ of the total lipids. Overall, the nutritional profile of Maltese Mushroom appears to be compatible with human consumption as described in traditional medicine [Cui et al., 2013; Jin et al., 2014], supporting the aim of this study.

TABLE 1. Nutritional information panel for lyophilized Maltese Mushroom. The data are for $100 \mathrm{~g}$ of the dried material.

\begin{tabular}{lc}
\hline Specification & Value \\
\hline Calories & $281 \mathrm{kcal}$ \\
Proteins & $1176 \mathrm{~kJ}$ \\
Total carbohydrate & $8.69 \mathrm{~g}$ \\
sugars & $45.5 \mathrm{~g}$ \\
Total fat & $29.6 \mathrm{~g}$ \\
saturated fat & $1.0 \mathrm{~g}$ \\
polyunsaturated fat & $27.36 \%$ \\
monounsaturated fat & $39.57 \%$ \\
Sodium & $33.07 \%$ \\
Total dietary fiber & $765 \mathrm{mg}$ \\
\hline
\end{tabular}

\section{Phenolic composition and antioxidant power}

Phenolic compounds are the principal plant constituent associated with antioxidant activity. Accordingly, their contents in the WP, EL and PP extracts were quantified first (Table 2). Based on the Folin-Ciocalteu assay results, the WP extract had a phenol concentration of approximately $3.3 \mathrm{mmol} / \mathrm{L}$ GAE (gallic acid equivalents) per gram of dry extract, which is on the same order of magnitude as that measured for other Cynomorium specimens [Jin et al., 2014; Rached et al., 2010; Wong et al., 2006] and for typical antioxidant plants (such as green tea, tomatoes, and oranges) [Cieślik et al., 2006]. The phenolic compounds were unevenly distributed between the EL and PP; their concentrations were higher (more than 2-fold) in the red-brown external layer. The flavonoid distribution also followed this trend, and anthocyanins were only detected in the EL. HPLC analysis revealed that cyanidin 3-O-glucoside accounted for the majority of the total anthocyanin content [Zucca et al., 2013a]. The UV-Vis spectra of both the WP and EL extracts supported this conclusion: two main absorption maxima were observed at $277 \mathrm{~nm}$ and $521 \mathrm{~nm}$. The addition of $5 \% \mathrm{AlCl}_{3}$ led to a $5 \mathrm{~nm}$ bathochromic shift in the visible band, which is consistent with the presence of $o r$ tho-hydroxyl groups on the cyanidin-3-O-glycoside B-ring [Delazar et al., 2010].

To fully assess the activities of the extracts, the total antioxidant power was determined by both the ET and HAT assays because only hydrogen atom transfer methods (such as ORAC-PYR) can evaluate the antioxidant capacity in addition to the reducing power [Zucca et al., 2010a]. The results are listed in Table 2. All the assays showed that the EL extract had a higher antioxidant power $(P<0.05$ for each method), indicating that the antioxidant capacity is mainly due to anthocyanins. However, both the ET and HAT assays showed that the PP extract also exhibited significant activity (on average, its antioxidant power was slightly less than half that of the EL extract), suggesting that the colorless component of Maltese Mushroom could also be used in nutraceutical formulations.

TABLE 2. Total antioxidant capacity of the Maltese Mushroom extracts from the external layer (EL), peeled plant (PP) and whole plant (WP). (n.d.) not detectable.

\begin{tabular}{lcccc}
\hline Assay & WP extract & EL extract & PP extract \\
\hline ORAC-PYR $(\mathrm{mTE} / \mathrm{g})$ & $6.81 \pm 0.41$ & $8.13 \pm 0.70$ & $2.39 \pm 0.24$ \\
$\mathrm{DPPH}(\mathrm{mTE} / \mathrm{g})$ & $1.16 \pm 0.04$ & $1.82 \pm 0.11$ & $0.76 \pm 0.03$ \\
$\mathrm{DPPH}\left(\mathrm{IC}_{50} \mu \mathrm{g} / \mathrm{mL}\right)$ & $10.70 \pm 0.39$ & $9.07 \pm 0.29$ & $25.09 \pm 1.54$ \\
$\mathrm{TEAC}(\mathrm{mTE} / \mathrm{g})$ & $3.86 \pm 0.23$ & $5.92 \pm 0.61$ & $1.83 \pm 0.10$ \\
TEAC $\left(\mathrm{IC}{ }_{50} \mu \mathrm{g} / \mathrm{mL}\right)$ & $188 \pm 40$ & $109 \pm 7$ & $266 \pm 86$ \\
FRAP $(\mathrm{mTE} / \mathrm{g})$ & $2.66 \pm 0.01$ & $3.54 \pm 0.02$ & $1.39 \pm 0.01$ \\
FRAP $(\mathrm{mmol} \mathrm{Fe} / \mathrm{g})$ & $3.12 \pm 0.01$ & $4.73 \pm 0.01$ & $2.37 \pm 0.01$ \\
Total phenolics $(\mathrm{mGAE} / \mathrm{g})$ & $3.34 \pm 0.01$ & $4.97 \pm 0.22$ & $2.06 \pm 0.02$ \\
Total flavonoids $(\mathrm{mCE} / \mathrm{g})$ & $1.12 \pm 0.01$ & $1.86 \pm 0.02$ & $0.87 \pm 0.01$ \\
$\begin{array}{l}\text { Total anthocyanin } \\
\text { (mg cyanidin }\end{array}$ & $6.6 \pm 0.3$ & $13.7 \pm 1.2$ & n.d. \\
3 - $O$-glucoside/g) & & & \\
\hline
\end{tabular}


Gallic acid was found to be one of the main components of the WP by HPLC analysis and was nearly equally distributed between the EL and PP [Zucca et al., 2013a]. These results indicated that gallic acid makes a significant contribution to the total antioxidant activity of the Maltese Mushroom extracts, which is consistent with several reports of the high antioxidant activity and healthy effects of gallic acid and suggests that it has potential for use in clinical and industrial applications [Jayamani \& Shanmugam, 2014].

TABLE 3. Fatty acid composition (\% of total fatty acids) determined by $\mathrm{GC}$ analysis of the lipid extracts obtained from the external layer (EL) and internal peeled part (PP) of Maltese Mushroom using the procedure of Folch et al. [1957].

\begin{tabular}{l|r|r}
\hline Fatty acids & EL extract & PP extract \\
\hline $12: 0$ & $0.34 \pm 0.11$ & $0.21 \pm 0.11$ \\
$14: 0$ & $1.28 \pm 0.27$ & $1.08 \pm 0.12$ \\
$16: 0$ & $25.02 \pm 2.49$ & $24.28 \pm 0.83$ \\
$16: 1 n-7$ & $1.96 \pm 0.62$ & $1.45 \pm 0.09$ \\
$18: 0$ & $4.34 \pm 1.06$ & $2.81 \pm 0.73$ \\
$18: 1 n-7$ & $1.41 \pm 0.14$ & $1.39 \pm 0.09$ \\
$18: 1 n-9$ & $21.70 \pm 1.27$ & $22.79 \pm 0.25$ \\
$18: 2 n-6$ & $33.34 \pm 4.17$ & $36.66 \pm 0.43$ \\
$18: 3 n-3$ & $3.84 \pm 1.20$ & $2.81 \pm 0.15$ \\
$20: 0$ & $0.43 \pm 0.21$ & $0.24 \pm 0.08$ \\
$20: 1 n-9$ & $5.51 \pm 0.53$ & $2.77 \pm 0.25$ \\
\hline SFA & $31.84 \pm 3.61$ & $28.69 \pm 1.42$ \\
MUFA & $30.05 \pm 1.38$ & $28.38 \pm 0.35$ \\
PUFA & $34.89 \pm 3.83$ & $39.47 \pm 0.29$ \\
\hline
\end{tabular}

SFA, saturated fatty acids; MUFA, monounsaturated fatty acids; PUFA polyunsaturated fatty acids. Mean and standard deviation of 4 samples.

\section{Composition of the Maltese Mushroom oil extracts}

Qualitative and quantitative information on the individual fatty acids in the Maltese Mushroom EL and PP oil extracts was obtained by GC analyses. The fatty acid compositions of the oil extracts, expressed as \% of the total fatty acids, are reported in Table 3 . The EL oil was composed of approximately $32 \%$ saturated fatty acids (SFA, mainly palmitic acid 16:0 and 18:0, 25 and 4\%, respectively), 30\% monounsaturated fatty acids (MUFA, mainly oleic acid 18:1 n-9 and 20:1, 22 and $5 \%$, respectively), and $35 \%$ polyunsaturated fatty acids (PUFA, mainly composed of the essential fatty acids linoleic acid 18:2 n-6 and a-linolenic acid 18:3 n-3, 33 and 4\%, respectively). The PP oil extract had a profile similar to that of the EL oil, with a higher level of PUFA (39\%) and lower amounts of MUFA (28\%) and SFA (29\%). It is interesting to note that both oils were characterized by a high proportion of PUFA. The fatty acid profile of the Maltese Mushroom oil obtained in this study differed from those obtained in previous studies [Rosa et al., 2012, 2015], possibly because the plant collection, extraction procedure, and plant metabolic activity were performed at a different time.

However, the striking similarities between the oil compositions of the different plant parts, PP and WP indicates that the whole plant could be used in nutraceutical applications.

\begin{abstract}
Antibacterial activity
Antibacterial activity is a crucial feature of plant extractbased formulations. Accordingly, the effects of the WP, PP and EL extracts on several Gram-positive and Gram-negative bacterial strains, including the clinical isolate $S$. aureus MRSA, were evaluated by the disc diffusion method. The results are summarized in Table 4.

Based on the parameters suggested by Alves and coworkers [Alves et al., 2000] (inhibition zones $<9 \mathrm{~mm}$, inactive; 9-12 $\mathrm{mm}$, less active; $13-18 \mathrm{~mm}$, active), the inhibition zones (1 mg/disc) of the aqueous Maltese Mushroom extracts against the Gram-positive strains, particularly those belonging to the Staphylococcus genera, were considerable. Of these
\end{abstract}

TABLE 4. Antibacterial activity of the aqueous Maltese Mushroom extracts determined by the disc diffusion method (inhibition zone in mm) (1 mg/ disc). Gentamicin and oxacilline were used as the reference antibiotic controls. (-) no activity.

\begin{tabular}{|c|c|c|c|c|}
\hline Bacteria & WP extract & EL extract & PP extract & Antibiotic control \\
\hline \multicolumn{5}{|c|}{ Gram positive } \\
\hline S. aureus DSM 1104 & $11.3 \pm 0.6$ & $11.0 \pm 0.1$ & $8.3 \pm 0.6$ & Ox $26.0 \pm 0.2$ \\
\hline S. epidermidis DSM 1798 & $12.3 \pm 0.6$ & $12.7 \pm 0.6$ & $10.7 \pm 0.6$ & Ox $23.0 \pm 0.1$ \\
\hline E. faecalis DSM 2570 & $9.3 \pm 0.6$ & $8.7 \pm 0.6$ & $7.7 \pm 0.6$ & Gent $12.5 \pm 0.6$ \\
\hline Staphylococcus MRSA & $10.3 \pm 0.6$ & $10.3 \pm 0.6$ & $10.0 \pm 0.1$ & - \\
\hline \multicolumn{5}{|c|}{ Gram negative } \\
\hline E. coli DSM 1103 & - & - & - & Gent $21.8 \pm 1.3$ \\
\hline E. cloacae DSM 30054 & - & - & - & Gent $19.5 \pm 0.6$ \\
\hline P. aeruginosa DSM 1117 & - & - & - & Gent $18.3 \pm 0.5$ \\
\hline A. baumannii DSM $30007^{\mathrm{T}}$ & $8.7 \pm 0.6$ & $8.3 \pm 0.6$ & - & Gent $16.5 \pm 1.0$ \\
\hline K. pneumoniae DSM 681 & - & - & - & Gent $21.5 \pm 1.0$ \\
\hline
\end{tabular}


bacterial strains, the clinical isolate MRSA $S$. aureus, a methicillin-resistant strain, is of particular clinical interest. These results are consistent with previous studies that showed that a crude acetone-water extract of $C$. songaricum had a moderate effect on a methicillin-resistant $S$. aureus strain [Jin et al., 2014]. Several mechanisms are known explaining the antimicrobial activity of anthocyanins, including interference with permeability of cell membranes and enzyme inhibition by their oxidized compounds [Marin et al., 2015]. However the effect of cyanidins on MRSA is actually quite controversial [Hatano et al., 2005], suggesting that the antimicrobial activity of Maltese Mushroom is not due to anthocyanins. This assumption is corroborated by the fact that the EL and PP extracts exhibited similar antibacterial activities for all the tested strains (Table 4), indicating that the antimicrobial activity might be due to gallic acid, which is consistent with previous reports [Kang et al., 2008] and well-known antimicrobial activity of gallates (possibly due to their prooxidant activity [Badhani et al., 2015]). Accordingly, the PP or WP should be preferred for microbiologically active formulations. Conversely, the extracts did not substantially inhibit the growth of Gram-negative strains. Only A. baumannii was inhibited by the WP and EL extracts, although it was not inhibited by the PP extract, strongly suggesting that anthocyanins are responsible for this specific biological activity.

\section{Anti-tyrosinase activity}

Tyrosinase (or polyphenol oxidase, PPO) is a copper monooxygenase involved in melanogenesis and food browning [Marongiu et al., 2007; Rescigno et al., 2011]. This enzyme catalyzes the hydroxylation of monophenols to $o$-diphenols and the oxidation of $o$-diphenols to $o$-quinones in the presence of molecular oxygen. It is desirable to inhibit this enzyme in food formulations to increase the shelf life of some food products, especially plant-based ones.

The WP, PP and EL extracts, even dialyzed samples, did not exhibit monophenolase or diphenolase activity for several well-known tyrosinase substrates [Olianas et al., 2005; Zucca et al., 2013b]. This finding supports the hypothesis that the PPO in plants is associated with the chloroplasts [Mayer,
2006]. It would be interesting to investigate whether the apparent absence of PPO corresponds to the absence of an encoding gene or to the lack of PPO gene expression.

Both the EL and PP extracts inhibited tyrosinase activity, although the EL extract was approximately twice as active as the PP extract $\left(\mathrm{IC}_{50}\right.$ of $\mathrm{EL}=30.4 \pm 1.0 \mu \mathrm{g}$, $\mathrm{IC}_{50}$ of $\mathrm{PP}=70.6 \pm 3.6 \mu \mathrm{g}$ ). It should be noted that the WP extract exhibited a stronger inhibition effect than both of its component extracts $\left(\mathrm{IC}_{50}=15.6 \pm 0.5 \mu \mathrm{g}\right)$, suggesting that secondary metabolites derived from interactions between the EL and PP play a crucial role in its anti-tyrosinase activity [Jin et al., 2014]. This finding is further supported by the $\mathrm{IC}_{50}$ value determined for the main EL component cyanidin-3-O-glycoside $(23.6 \pm 2.4 \mu \mathrm{g})$, which suggests that this compound is only responsible for a small fraction of the global anti-tyrosinase activity.

Overall, the anti-tyrosinase activity data suggest that the WP is a possible candidate for use in food formulations to prevent browning.

\section{Cytotoxic effects of the aqueous extracts on B16F10 cells}

Melanoma is the most malignant skin cancer, and its occurrence has remarkably increased over the past few decades. The B16F10 mouse melanoma cell line is highly metastatic and is commonly used to screen antitumor agents. The cytotoxicity of the aqueous Maltese Mushroom extracts against the B16F10 cells were tested (MTT assay) and compared. Figure 2 shows the viability, expressed as \% of the control, of the B16F10 cells after incubation in the presence of different concentrations of the EL, PP, and WP extracts (25-500 $\mu \mathrm{g} / \mathrm{mL}$ ) for $24 \mathrm{~h}$. This concentration range was chosen based on similar experiments performed with other plant extracts. Treatment with a $100 \mu \mathrm{g} / \mathrm{mL}$ EL extract solution significantly reduced the cell viability compared to the control, and the viability was $49 \%$ at EL extract concentrations of 250 and $500 \mu \mathrm{g} / \mathrm{mL}$. The cell viability decreased by $41 \%$ and $68 \%$ when they were treated with 250 and $500 \mu \mathrm{g} / \mathrm{mL} \mathrm{WP}$ extract solutions, respectively. Although the PP extract was less effective than the EL and WP extracts, it reduced the cell viability by $46 \%$ at a concentration of $500 \mu \mathrm{g} / \mathrm{mL}$. The poten-

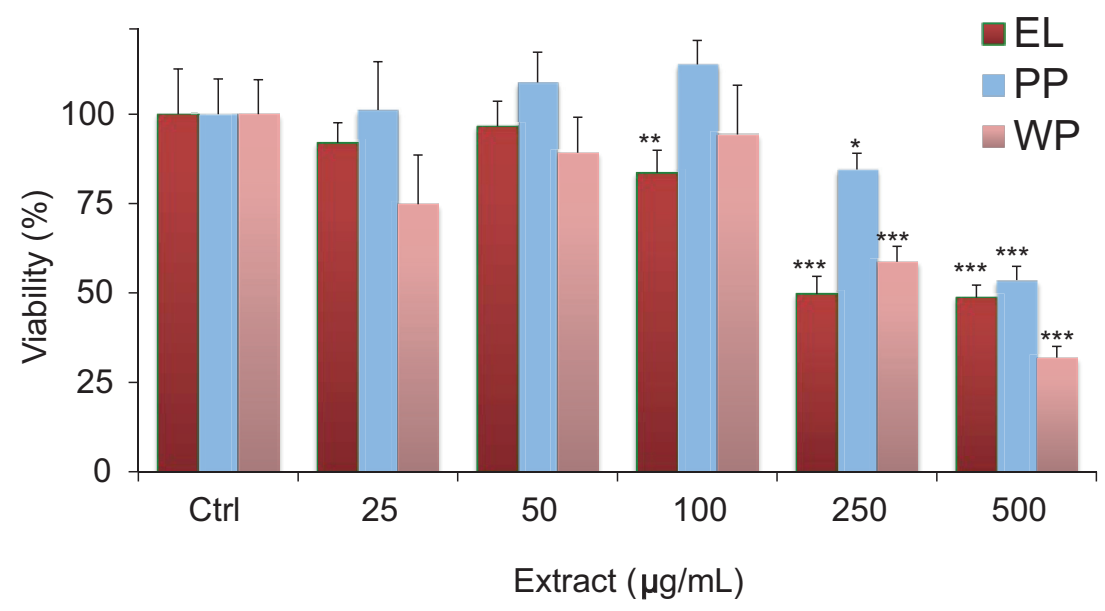

FIGURE 2. Viability (expressed as \% of the control) (MTT assay) after incubating melanoma B16F10 cells with the Maltese Mushroom extracts obtained from the external layer (EL), internal peeled part (PP), and whole plant (WP). ${ }^{* * *}=P<0.001 ;{ }^{* *}=P<0.01{ }^{*}{ }^{*}=P<0.05$ vs control; a' $=P<0.001$ vs control for both cell lines. 
tial anti-cancer properties of natural extracts obtained from plants/herbs used in traditional medicine have received much attention. Evaluating the cytotoxic activities of the aqueous Maltese Mushroom extracts revealed their ability to reduce the viability of B16F10 cancer cells. Recently, gallic acid was shown to induce cellular apoptosis in B16F10 melanoma cells [Liu et al., 2014], probably due to the complex prooxidant action of gallate compounds [Badhani et al., 2015]. A proteomic approach revealed that the gallic acid-induced cellular apoptosis in these cells is associated with metabolic glycolysis. Therefore, the cytotoxic effect observed in this study could be attributed to gallic acid because it is one of the major components in the Maltese Mushroom extracts.

In a previous study, the fixed oil obtained from the whole plant by SFE extraction also significantly inhibited the growth of melanoma and colon cancer cells, thus demonstrating the potential of Maltese Mushroom [Rosa et al., 2015].

Interestingly, the extracts did not affect the cells' ability to produce melanin.

\section{Pro-erectile activity in adult male rats}

Many studies of $C$. songaricum have shown that its extracts affect the reproductive system; therefore, many products containing these extracts have been marketed for the treatment of erectile dysfunction, premature ejaculation, spermatorrhea and general sexual health [Cui et al., 2013]. Only a few studies of the effects of Maltese Mushroom on the reproductive system have appeared in the literature. Abd El-Rahman and colleagues found that a plant water extract induced a significant increase in the sperm count of rats, increased the percentage of live sperm and their motility and decreased the number of abnormal sperm [Abd El-Rahman et al., 1999].

A synergistic combination of an aqueous Withania somnifera extract and Maltese Mushroom extract was found to have a direct spermatogenic influence on the seminiferous tubules of immature rats, presumably by exerting a testosterone-like effect [Abdel-Magied et al., 2001].

To our knowledge, the effect of Maltese Mushroom extracts on penile erection in laboratory animals or humans has not been studied. Our results show for the first time that aqueous Maltese Mushroom extracts of the whole plant exhibited pro-erectile activity when administered subcutaneously in adult male rats (see Figure 3). Because the pro-erectile activity persisted after the colored component(s) were removed from the extract and the colored component(s) did not exhibit pro-erectile activity, it was concluded that the pro-erectile activity was due to one or more colorless, highly hydrophilic components, which might be present in both the internal and external parts of the plant and whose structures are currently unknown. Indeed, the plant components that have been identified (i.e., gallic acid, cyanidin 3-O-glucoside, flavonoids, etc.) have not been reported to exhibit pro-erectile activity, although they exert a general protective effect on penile tissues due to their antioxidant activity [Cui et al., 2013]. Approximately $10 \mathrm{mg}$ of the WP extract exhibited a pro-erectile activity similar to that induced by $20 \mu \mathrm{g}$ of apomorphine, a dopamine receptor agonist well known for its ability to induce penile erection in laboratory animals and humans by acting sys-
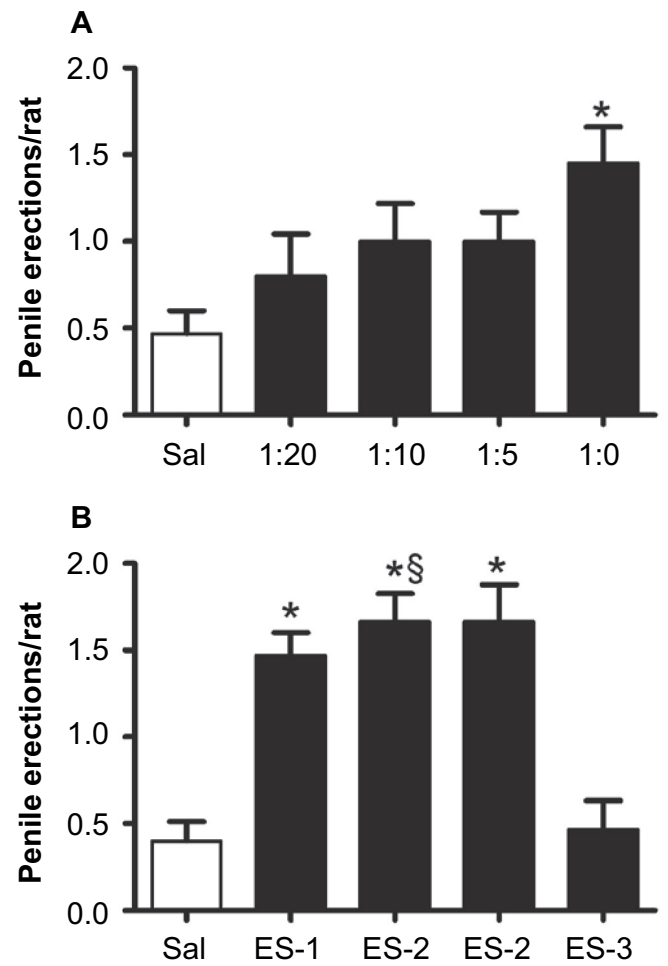

FIGURE 3. Effect of Maltese Mushroom extract solutions 1, 2 and 3 on penile erection in male rats. ES-1 (1:0) and its dilutions $(1: 5 ; 1: 10 ; 1: 20)$ (A), ES-2 and ES-3 (B) were administered SC in aliquots of $0.1 \mathrm{~mL} / 80 \mathrm{~g}$ of rat body weight. At the doses used, no gross behavioral changes were observed. ${ }^{*} P<0.01$ with respect to saline-treated rats (one-way ANOVA followed by Tukey's multicomparison test). § This group was treated with $0.05 \mathrm{~mL}$ of ES-2 instead of $0.1 \mathrm{~mL} / 80 \mathrm{~g}$ rat body weight.

temically [Argiolas, 2005; Sanna et al., 2011]. It is currently unknown whether the Maltese Mushroom extracts induced penile erection by acting locally (i.e., in the penile tissues), systemically or both. These results are consistent with a US patent claiming that a $C$. songarium $\mathrm{L}$. Rupr. extract exhibits anti-phosphodiesterase activity, potentiating the relaxation of isolated cavernous smooth muscle strips by cGMP, which makes the extract suitable to treat erectile dysfunction (US Patent Ref. No. US20030157208 A1 [Wang et al., 2003]).

\section{SUMMARY AND CONCLUSIONS}

In the past, medicinal properties of Maltese Mushroom were held in high regard [Dharmananda, 2011]. As time passed, its use in traditional medicine waned except in some populations of the Arabian peninsula.

In this study, some healthful properties of Maltese Mushroom were verified, and other biological activities of this plant (cytotoxicity against B16F10 mouse melanoma cells and anti-tyrosinase, antibacterial, and pro-erectile activities) were described for the first time. Despite being results about only partially differentiated plant materials, needing some additional in-depth analysis, the reported data highlight the potential of Maltese Mushroom as a functional food. Accordingly, additional studies of toxicity in normal cell lines and in vivo model will be necessary prior to human application. In fact, therapeutic effects of plant extracts could 
be associated with the presence of substances present in low amount in the extract or synergistic performance of many components.

\section{RESEARCH FUNDING}

This work was partially supported by grants from the Università di Cagliari (Contributo di Ateneo per la Ricerca, C.A.R. 2012-13-14) for PZ, AA, and AR.

\section{REFERENCES}

1. Abd El-Rahman H.A., El-Badry A.A., Mahmoud O.M., Harraz F.A., The effect of the aqueous extract of Cynomorium coccineum on the epididymal sperm pattern of the rat. Phytother. Res., 1999, $13,3,248-250$.

2. Abdel-Magied E.M., Abdel-Rahman H.A., Harraz F.M., The effect of aqueous extracts of Cynomorium coccineum and Withania somnifera on testicular development in immature Wistar rats. J. Ethnopharmacol., 2001, 75, 1, 1-4.

3. Alves T.M. d.A., Silva A.F., Brandão M., Grandi T.S.M., Smânia E.d. F.A., Smânia Júnior A., Zani C.L., Biological screening of Brazilian medicinal plants. Mem. Inst. Oswaldo Cruz, 2000, 95, 367-373.

4. Argiolas A., Male erectile dysfunction: Chemical pharmacology of penile erection. Drug Discovery Today: Therapeutic Strategies, 2005, 2, 1, 31-36.

5. Badhani B., Sharma N., Kakkar R., Gallic acid: a versatile antioxidant with promising therapeutic and industrial applications. RSC Advances, 2015, 5, 35, 27540-27557.

6. Chahmi N., Anissi J., Jennan S., Farah A., Sendide K., El Hassouni M., Antioxidant activities and total phenol content of Inula viscosa extracts selected from three regions of Morocco. Asian Pac. J. Trop. Biomed., 2015, 5, 3, 228-233.

7. Cieślik E., Gręda A., Adamus W., Contents of polyphenols in fruit and vegetables. Food Chem., 2006, 94, 1, 135-142.

8. Cui Z., Guo Z., Miao J., Wang Z., Li Q., Chai X., Li M., The genus Cynomorium in China: An ethnopharmacological and phytochemical review. J. Ethnopharmacol., 2013, 147, 1, 1-15.

9. Delazar A., Khodaie L., Afshar J., Nahar L., Sarker S.D., Isolation and free-radical-scavenging properties of cyanidin 3-O-glycosides from the fruits of Ribes biebersteinii Berl. Acta Pharm., 2010, 60, 1, 1-11.

10. Dharmananda S., Cynomorium - Parasitic plant widely used in traditional medicine (Accessed June 18th 2015, [http://www. itmonline.org/arts/cynomorium.htm)]. 2011.

11. Duke J.A., Duke's Handbook of Medicinal Plants of the Bible. C. P.-T. F. Group. 2008, Boca Raton (FL), p. 528.

12. Folch J., Lees M., Sloane Stanley G.H., A simple method for the isolation and purification of total lipides from animal tissues. J. Biol. Chem., 1957, 226, 1, 497-509.

13. Gonçalves M.J., Piras A., Porcedda S., Marongiu B., Falconieri D., Cavaleiro C., Rescigno A., Rosa A., Salgueiro L., Antifungal activity of extracts from Cynomorium coccineum growing wild in Sardinia island (Italy). Nat. Prod. Res., 2015, 29, 2247-2250.

14. Hatano T., Kusuda M., Inada K., Ogawa T.O., Shiota S., Tsuchiya T., Yoshida T., Effects of tannins and related polyphenols on methicillin-resistant Staphylococcus aureus. Phytochemistry, 2005, 66, 17, 2047-2055.
15. Huang D., Ou B.X., Prior R.L., The chemistry behind antioxidant capacity assays. J. Agric. Food Chem., 2005, 53, 6, 1841-1856.

16. Institute C.a.L.S., Performance standards for antimicrobial disk susceptibility testing. Approved standard. 2012, Eleventh edition. NCCLS document M02-A11. p. 32.

17. Jayamani J., Shanmugam G., Gallic acid, one of the components in many plant tissues, is a potential inhibitor for insulin amyloid fibril formation. Eur. J. Med. Chem., 2014, 85, 352-358.

18. Jin S.W., Chen G.L., Du J.J. M., Wang L.H., An T.Y., Antioxidant properties and principal phenolic composition of Cynomorium songaricum Rupr. Int. J. Food Prop., 2014, 17, 13-25.

19. Kang M.S., Oh J.S., Kang I.C., Hong S.J., Choi C.H., Inhibitory effect of methyl gallate and gallic acid on oral bacteria. J. Microbiol., 2008, 46, 6, 744-750.

20. Liu C., Lin J.-J., Yang Z.-Y., Tsai C.-C., Hsu J.-L., Wu Y.-J., Proteomic study reveals a co-occurrence of gallic acid-induced apoptosis and glycolysis in B16F10 melanoma cells. J. Agric. Food Chem., 2014, 62, 48, 11672-11680.

21. Marin L., Miguelez E.M., Villar C.J., Lombo F., Bioavailability of dietary polyphenols and gut microbiota metabolism: antimicrobial properties. BioMed Res. Int., 2015, 2015, article ID 905215.

22. Marongiu B., Piras A., Porcedda S., Tuveri E., Sanjust E., Meli M., Sollai F., Zucca P., Rescigno A., Supercritical $\mathrm{CO}_{2}$ extract of Cinnamomum zeylanicum: Chemical characterization and antityrosinase activity. J. Agric. Food Chem., 2007, 55, 24, 10022$-10027$.

23. Mayer A.M., Polyphenol oxidases in plants and fungi: going places? A review. Phytochemistry, 2006, 67, 21, 2318-2331.

24. Meng H.-C., Wang S., Li Y., Kuang Y.-Y., Ma C.-M., Chemical constituents and pharmacologic actions of Cynomorium plants. Chin. J. Nat. Med., 2013, 11, 4, 321-329.

25. Mulabagal V., Keller W.J., Calderón A.I., Quantitative analysis of anthocyanins in Euterpe oleracea (açaí) dietary supplement raw materials and capsules by Q-TOF liquid chromatography/ mass spectrometry. Pharm. Biol., 2012, 50, 10, 1289-1296.

26. Olianas A., Sanjust E., Pellegrini M., Rescigno A., Tyrosinase activity and hemocyanin in the hemolymph of the slipper lobster Scyllarides latus. J. Comp. Physiol. B., 2005, 175, 6, 405-411.

27. Rached W., Benamar H., Bennaceur M., Marouf A., Screening of the antioxidant potential of some Algerian indigenous plants. J. Biol. Sci., 2010, 10, 4, 316-324.

28. Rescigno A., Casañola-Martin G.M., Sanjust E., Zucca P., Marrero-Ponce Y., Vanilloid derivatives as tyrosinase inhibitors driven by virtual screening-based QSAR models. Drug Test. Anal., 2011, 3, 3, 176-181.

29. Rescigno A., Sanjust E., Pedulli G.F., Valgimigli L., Spectrophotometric method for the determination of polyphenol oxidase activity by coupling of 4-tert-butyl-o-benzoquinone and 4-amino-N,N-diethylaniline. Anal. Lett., 1999, 32, 10, 2007-2017.

30. Rescigno A., Zucca P., Flurkey A., Inlow J., Flurkey W.H., Identification and discrimination between some contaminant enzyme activities in commercial preparations of mushroom tyrosinase. Enzyme Microb. Technol., 2007, 41, 5, 620-627.

31. Roncaglioni M.C., Tombesi M., Avanzini F., Barlera S., Caimi V., Longoni P., Marzona I., Milani V., Silletta M.G., Tognoni G., Marchioli R., n-3 fatty acids in patients with multiple cardiovascular risk factors. N. Engl. J. Med., 2013, 368, 19, 1800-1808. 
32. Rosa A., Nieddu M., Piras A., Atzeri A., Putzu D., Rescigno A., Maltese mushroom (Cynomorium coccineum L.) as source of oil with potential anticancer activity. Nutrients, 2015, 7, 2, 849-864.

33. Rosa A., Rescigno A., Piras A., Atzeri A., Scano P., Porcedda S., Zucca P., Assunta Dessì M., Chemical composition and effect on intestinal Caco-2 cell viability and lipid profile of fixed oil from Cynomorium coccineum L. Food Chem. Toxicol., 2012, 50, 10, 3799-3807.

34. Sanjust E., Mocci G., Zucca P., Rescigno A., Mediterranean shrubs as potential antioxidant sources. Nat. Prod. Res., 2008, 22, 8, 689-708.

35. Sanna F., Succu S., Hübner H., Gmeiner P., Argiolas A., Melis M. R., Dopamine D2-like receptor agonists induce penile erection in male rats: Differential role of D2, D3 and D4 receptors in the paraventricular nucleus of the hypothalamus. Behav. Brain Res., 2011, 225, 1, 169-176.

36. Sanna F., Succu S., Melis M.R.,Argiolas A., Dopamine agonistinduced penile erection and yawning: Differential role of D 2-like receptor subtypes and correlation with nitric oxide production in the paraventricular nucleus of the hypothalamus of male rats. Behav. Brain Res., 2012, 230, 2, 355-364.

37. Schiller C.D., Kainz A., Mynett K., Gescher A., Assessment of viability of hepatocytes in suspension using the MTT assay. Toxicol. In Vitro, 1992, 6, 6, 575-578.

38. Shi G., Jiang W., Cai L., Sui G., Molecular characteristics and antitumor capacity of glycan extracted from Cynomorium songaricum. Int. J. Biol. Macromol., 2011, 48, 5, 788-792.

39. Soddu G., Sanjust E., Murgia S., Rescigno A., Interference of some tryptophan metabolites in the formation of melanin in vitro. Pigment Cell Res., 2004, 17, 2, 135-141.

40. Sollai F., Zucca P., Sanjust E., Steri D., Rescigno A., Umbelliferone and esculetin: Inhibitors or substrates for polyphenol oxidases? Biol. Pharm. Bull., 2008, 31, 12, 2187-2193.

41. Traka M.H., Mithen R.F., Plant science and human nutrition: Challenges in assessing health-promoting properties of phytochemicals. Plant Cell, 2011, 23, 7, 2483-2497.

42. Wang J., Zhang J., Zhao B., Wu Y., Wang C., Wang Y., Structural features and hypoglycaemic effects of Cynomorium songaricum polysaccharides on STZ-induced rats. Food Chem., 2010, 120, 2, 443-451.
43. Wang R., Huang Y., Zhao W., Cynomoriaceae extract and use in treating erectile dysfunction. Google Patents. P. S. IFI Claims. 2003.

44. Wong C.C., Li H.B., Cheng K.W., Chen F., A systematic survey of antioxidant activity of 30 Chinese medicinal plants using the ferric reducing antioxidant power assay. Food Chem., 2006, 97, 705-711.

45. Yang W.M., Kim H.Y., Park S.Y., Kim H.M., Chang M.S., Park S.K., Cynomorium songaricum induces spermatogenesis with glial cell-derived neurotrophic factor (GDNF) enhancement in rat testes. J. Ethnopharmacol., 2010, 128, 3, 693-696.

46. Yu F.R., Liu Y., Cui Y.Z., Chan E.Q., Xie M.R., McGuire P.P., Yu F.H., Effects of a flavonoid extract from Cynomorium songaricum on the swimming endurance of rats. Am. J. Chin. Med., 2010, 38, 1, 65-73.

47. Zucca P., Rescigno A., Olianas A., MacCioni S., Sollai F.A., Sanjust E., Induction, purification, and characterization of a laccase isozyme from Pleurotus sajor-caju and the potential in decolorization of textile dyes. J. Mol. Catal. B: Enzym., 2011, 68, 2, 216-222.

48. Zucca P., Rosa A., Tuberoso C.I.G., Piras A., Rinaldi A.C., Sanjust E., Dessì M.A., Rescigno A., Evaluation of antioxidant potential of "Maltese mushroom" (Cynomorium coccineum) by means of multiple chemical and biological assays. Nutrients, 2013a, 5, 1, 149-161.

49. Zucca P., Sanjust E., Loi M., Sollai F., Ballero M., Pintus M., Rescigno A., Isolation and characterization of polyphenol oxidase from Sardinian poisonous and non-poisonous chemotypes of Ferula communis (L.). Phytochemistry, 2013b, 90, 16-24.

50. Zucca P., Sanjust E., Trogu E., Sollai F., Rescigno A., Evaluation of antioxidant capacity of antioxidant-declared beverages marketed in Italy. It. J. Food Sci., 2010a, 22, 3, 313-319.

51. Zucca P., Vinci C., Rescigno A., Dumitriu E., Sanjust E., Is the bleaching of phenosafranine by hydrogen peroxide oxidation catalyzed by silica-supported 5,10,15,20-tetrakis(sulfonatophenyl)porphine-Mn(III) really biomimetic? J. Mol. Catal. A: Chem., 2010b, 321, 1-2, 27-33.

Submitted: 4 August 2015. Revised: 25 January and 18 February 2016. Accepted: 30 March 2016. Published on-line: 10 June 2016. 